\title{
Geometry of homogeneous polynomials on non symmetric convex bodies
}

\author{
Gustavo A. Muñoz-Fernández* Szilárd Gy. Révész ${ }^{\dagger}$ \\ Juan B. Seoane-Sepúlveda
}

\begin{abstract}
If $\Delta$ stands for the region enclosed by the triangle in $\mathbb{R}^{2}$ of vertices $(0,0)$, $(0,1)$ and $(1,0)$ (or simplex for short), we consider the space $\mathcal{P}\left({ }^{2} \Delta\right)$ of the 2-homogeneous polynomials on $\mathbb{R}^{2}$ endowed with the norm given by $\| a x^{2}+$ $b x y+c y^{2} \|_{\Delta}:=\sup \left\{\left|a x^{2}+b x y+c y^{2}\right|:(x, y) \in \Delta\right\}$ for every $a, b, c \in \mathbb{R}$. We investigate some geometrical properties of this norm. We provide an explicit formula for $\|\cdot\|_{\Delta}$, a full description of the extreme points of the corresponding unit ball and a parametrization and a plot of its unit sphere. Using this geometrical information we also find sharp Bernstein and Markov inequalities for $\mathcal{P}\left({ }^{2} \Delta\right)$ and show that a classical inequality of Martin does not remain true for homogeneous polynomials on non symmetric convex bodies.
\end{abstract}

\section{Introduction and notation.}

Let us recall first that, given a convex body $C$ in a Banach space, a point $e \in C$ is said to be extreme if $x, y \in C$ and $\lambda x+(1-\lambda) y=e$, for some $0<\lambda<1$, entails $x=y=e$. Equivalently, $e \in C$ is extreme if and only if $C \backslash\{e\}$ is convex. The importance of having a good description of the extreme points of a convex body rests on the fact that the Krein-Milman Theorem lets us characterize the convex body itself using its extreme points.

In the last few years the study of the extreme points in the unit ball of finite dimensional spaces of polynomials has become a fruitful research field. Konheim and Rivlin [16] found a characterization of the extreme polynomials of the unit ball of the space of polynomials in one real variable endowed with the sup norm on $[-1,1]$. In $[1,20]$ the authors describe the geometry of the spaces of trinomials on the real line with the sup norm on symmetric intervals. In a similar direction, Choi and Kim [5, 6, 7] considered the same problem for scalar-valued 2-homogeneous polynomials on the real spaces $\ell_{1}^{2}, \ell_{2}^{2}$ and $\ell_{\infty}^{2}$ whereas Grecu [10] treated the same

*Supported by MTM 2006-03531.

†Supported in part by Hungarian National Foundation for Scientific Research, Grant \# T049301.

${ }^{\ddagger}$ Supported by MTM 2006-03531. 
question for scalar-valued 2-homogeneous polynomials on the real spaces $\ell_{p}^{2}$ with $1<p<\infty$. See also $[8,9,10,11,12,13]$ for related questions concerning real or complex homogeneous polynomials of degree 2 or 3 . Trigonometric trinomials (real or complex) have also been studied by Aron and Klimek [1], Neuwirth [23] and the second author [24]. This paper introduces a novelty, never studied before, by presenting a full description of the extreme points of a space of 2-homogeneous polynomials defined on a non symmetric convex body. To be more specific, we study here the geometry of the unit ball of the space of 2-homogeneous polynomials on $\mathbb{R}^{2}$ endowed with the norm defined by

$$
\|P\|_{\Delta}=\sup \{|P(\mathbf{x})|: \mathbf{x} \in \Delta\}
$$

where $\Delta$ represents the region enclosed by the triangle in $\mathbb{R}^{2}$ of vertices $(0,0),(0,1)$ and $(1,0)$ (or simplex for short). From now on we will represent the space described just above by $\mathcal{P}\left({ }^{2} \Delta\right)$. If $L \in \mathcal{L}\left({ }^{2} \mathbb{R}^{2}\right)$, we also define

$$
\|L\|_{\Delta}=\sup \{|L(\mathbf{x}, \mathbf{y})|: \mathbf{x}, \mathbf{y} \in \Delta\}
$$

The mapping that assigns to every $(a, b, c) \in \mathbb{R}^{3}$ the polynomial $P(x, y)=a x^{2}+$ $b x y+c y^{2}$ lets us identify $\mathcal{P}\left({ }^{2} \Delta\right)$ with $\left(\mathbb{R}^{3},\|\cdot\|_{\Delta}\right)$, where $\|(a, b, c)\|_{\Delta}=\|P\|_{\Delta}$. This latter representation together with the usual polynomial representation of $\mathcal{P}\left({ }^{2} \Delta\right)$ will be interchanged throughout the paper.

A full description of the extreme points of a convex body has many other applications. For instance, if the extreme points of the unit ball of a space of polynomials are known, the problem of obtaining sharp inequalities where norms of polynomials are compared may be simplified by using the well-known fact that a convex function (like a polynomial norm, for instance) defined on a convex body (like the unit ball of a finite dimensional polynomial space) attains its maximum at one extreme point of the convex body. From now on we will refer to this method as the Krein-Milman Approach. In this paper we apply this method in order to obtain sharp Bernstein and Markov type inequalities for polynomials in $\mathcal{P}\left({ }^{2} \Delta\right)$. Using other techniques, Bernstein and Markov type inequalities for polynomials on triangles have also been studied in e.g. [18, 22], however sharpness of these estimates cannot be seen from these papers. Bernstein and Markov type inequalities for non-homogeneous polynomials on arbitrary normed spaces are considered in many papers, see e.g. [2, 14, 19, 25, 26] and the references therein. These results, however, make essential use of the fact that the unit ball of a normed space is a symmetric convex body, which is not the case for the simplex.

The Krein-Milman Approach has also been used in [21] in order to obtain sharp Bernstein and Markov type inequalities for trinomials on the real line. In fact the Krein-Milman approach has been used repeatedly in the literature. Konheim and Rivlin [16], and much earlier, Voronovskaya [27], can be considered pioneers of this method.

There is another question that arises from the study of Markov inequalities for homogeneous polynomials on a Banach space that is treated in Section 4. If $E$ is a Banach space over the filed $\mathbb{K}=\mathbb{R}$ or $\mathbb{C}$ with closed unit ball $\mathrm{B}_{E}$ and $n \in \mathbb{N}$, then 
we will denote by $\mathcal{P}\left({ }^{n} E\right)$ and $\mathcal{L}^{s}\left({ }^{n} E\right)$ the space of all continuous $n$-homogeneous polynomials on $E$ and the space of all continuous symmetric $n$-linear forms on $E^{n}$ endowed respectively with the norms given by

$$
\begin{aligned}
\|P\| & =\sup \left\{\|P(x)\|: x \in \mathrm{B}_{E}\right\}, \\
\|L\| & =\sup \left\{\left\|L\left(x_{1}, \ldots, x_{n}\right)\right\|: x_{1}, \ldots, x_{n} \in \mathrm{B}_{E}\right\} .
\end{aligned}
$$

According to an old and well-known algebraic result, for every $P \in \mathcal{P}\left({ }^{n} E\right)$ there exists a unique $\check{P} \in \mathcal{L}^{s}\left({ }^{n} E\right)$ such that $P(x)=\check{P}\left(x, .{ }^{n}, x\right)$, for every $x \in E$ and the mapping $\mathcal{P}\left({ }^{n} E\right) \ni P \mapsto \check{P} \in \mathcal{L}^{s}\left({ }^{n} E\right)$ is an algebraic isomorphism. Furthermore, Martin [17] proved that

$$
\|P\| \leq\|\check{P}\| \leq \frac{n^{n}}{n !}\|P\|,
$$

for every $P \in \mathcal{P}\left({ }^{n} E\right)$. It can be shown that the constant $\frac{n^{n}}{n !}$ cannot be improved in general since equality is achieved for $\ell_{1}^{n}$ and the polynomial defined by $\Phi\left(x_{1}, \ldots, x_{n}\right)=x_{1} \cdots x_{n}$ for every $\left(x_{1}, \ldots, x_{n}\right) \in \mathbb{K}^{n}$. However, for an specific space $E$ the constant $\frac{n^{n}}{n !}$ in (1) can be improved. The best constant $\mathbb{K}(n ; E)$ in the inequality

$$
\|\check{P}\| \leq M\|P\|
$$

for every $P \in \mathcal{P}\left({ }^{n} E\right)$ is called the $n$th polarization constant of $E$.

In Section 4 we give a version of (1) for polynomials on $\Delta$, showing that in this case

$$
\|\check{P}\|_{\Delta} \leq 3\|P\|_{\Delta}
$$

for every $P \in \mathcal{P}\left({ }^{2} \Delta\right)$ and that 3 is optimal. This shows that (1) does not hold for polynomials on a non symmetric convex body. The analogous question of a sharp Bernstein inequality for the totality of all degree 2 polynomials is a more difficult question, which, on the other hand, would have some interest in itself [3]. However, the complexity of the description of the set of extreme polynomials definitely exceeds that of the current work, and at the moment we do not see a full solution of this situation.

From now on, $\mathrm{S}_{\Delta}$ and $\mathrm{B}_{\Delta}$ will denote, respectively, the unit sphere and unit ball of the space $\mathcal{P}\left({ }^{2} \Delta\right)$. If $C$ is a convex body, $\operatorname{ext}(C)$ will denote the set of extreme points of $C$. Also, $\pi_{a b}$ will denote the linear projection given by $\pi_{a b}(a, b, c)=(a, b)$, for every $(a, b, c) \in \mathbb{R}^{3}$. The plots appearing in this paper were produced using Maple (see, e.g. [15]).

\section{The unit ball of $\mathcal{P}\left({ }^{2} \Delta\right)$.}

First of all we find a formula for $\|\cdot\|_{\Delta}$ in the following result.

Theorem 2.1 Let $a, b, c \in \mathbb{R}$ and $P(x, y)=a x^{2}+b x y+c y^{2}$. Then

$$
\|P\|_{\Delta}= \begin{cases}\max \left\{|a|,|c|,\left|\frac{b^{2}-4 a c}{4(a-b+c)}\right|\right\} & \text { if } a-b+c \neq 0 \text { and } 0<\frac{2 c-b}{2(a-b+c)}<1, \\ \max \{|a|,|c|\} & \text { otherwise. }\end{cases}
$$


Proof. If we let $P(x, y)=a x^{2}+b x y+c y^{2}$, then the norm of $P$ is attained either in $\partial \Delta$, or at a critical point of $P$ in $\stackrel{\circ}{\Delta}$. Let us evaluate first the maximum of $P$ over $\partial \Delta$. To start with, notice that the maximum of $|P|$ over the side $\{(t, 1-t): t \in[0,1]\}$ coincides with the norm of the polynomial $p(t)=(a-b+c) t^{2}+(b-2 c) t+c$ over the interval $[0,1]$. Performing some calculations, it can be proved that

$$
\|p\|_{[0,1]}= \begin{cases}\max \left\{|a|,|c|,\left|\frac{b^{2}-4 a c}{4(a-b+c)}\right|\right\} & \text { if } a-b+c \neq 0 \text { and } 0<\frac{2 c-b}{2(a-b+c)}<1, \\ \max \{|a|,|c|\} & \text { otherwise. }\end{cases}
$$

On the other hand the maxima of $|P|$ over the sides $\{(t, 0): t \in[0,1]\}$ and $\{(0, t)$ : $t \in[0,1]\}$ are $|a|$ and $|c|$ respectively. Since $\max \{|a|,|c|\} \leq\|p\|_{[0,1]}$, we obviously have that $\|P\|_{\partial \Delta}=\|p\|_{[0,1]}$.

As for the critical points of $P$ in $\stackrel{\circ}{\Delta}$, notice that in case $P$ has a critical point $(x, y)$ other than $(0,0)$ then the restriction of $P$ to the line $L(t):=\{(t x, t y): t \in \mathbb{R}\}$, always having the form $C t^{2}$ on $L$, must necessarily have $C=0$, which implies that $P(x, y)=0$, and the critical point in question does not qualify for an extrema (unless $P \equiv 0)$.

Hence, $\|P\|_{\Delta}=\|P\|_{\partial \Delta}=\|p\|_{[0,1]}$. This concludes the proof.

In order to parametrize $S_{\Delta}$ it will be useful to know what the projection of $S_{\Delta}$ onto any of the coordinate planes looks like.

Theorem 2.2 The projection of $\mathrm{S}_{\Delta}$ onto the ac-plane is $\mathrm{B}_{\ell_{\infty}^{2}}$.

Proof. If we take $(a, b, c) \in \mathrm{S}_{\Delta}$ then by (2) it follows that $\|(a, c)\|_{\infty} \leq\|(a, b, c)\|_{\Delta}=$ 1. Therefore $\pi_{a c}\left(\mathrm{~S}_{\Delta}\right) \subseteq \mathrm{B}_{\ell_{\infty}^{2}}$. In order to conclude the proof it will be enough to show that $\mathrm{S}_{\ell_{\infty}^{2}} \subseteq \pi_{a c}\left(\mathrm{~S}_{\Delta}\right)$. But this is easy to check. Indeed, if $(a, c) \in \mathrm{S}_{\ell_{\infty}^{2}}$, by (2) we have that

$$
\|(a, 0, c)\|_{\Delta}= \begin{cases}\max \left\{1,\left|\frac{a c}{a+c}\right|\right\} & \text { if } a+c \neq 0 \text { and } 0<\frac{c}{a+c}<1, \\ 1 & \text { otherwise, }\end{cases}
$$

and since $\left|\frac{a c}{a+c}\right|<|a| \leq 1$ whenever $a+c \neq 0$ and $0<\frac{c}{a+c}<1$, it follows that $\|(a, 0, c)\|_{\Delta}=1$. Therefore $(a, c)=\pi_{a c}(a, 0, c) \in \pi_{a c}\left(\mathrm{~S}_{\Delta}\right)$.

With the help of Theorems 2.1 and 2.2, now it is possible to characterize the extreme points of $\mathrm{B}_{\Delta}$.

Theorem 2.3 If we define the mappings

$$
f_{+}(a, c)=2+2 \sqrt{(1-a)(1-c)}
$$

and

$$
f_{-}(a, c)=-f_{+}(-a,-c)=-2-2 \sqrt{(1+a)(1+c)},
$$

for every $(a, c) \in \mathrm{B}_{\ell_{\infty}^{2}}$ and the set

$$
F=\left\{(a, b, c) \in \mathbb{R}^{3}:(a, c) \in \mathrm{S}_{\ell_{\infty}^{2}} \text { and } f_{-}(a, c) \leq b \leq f_{+}(a, c)\right\},
$$

then 


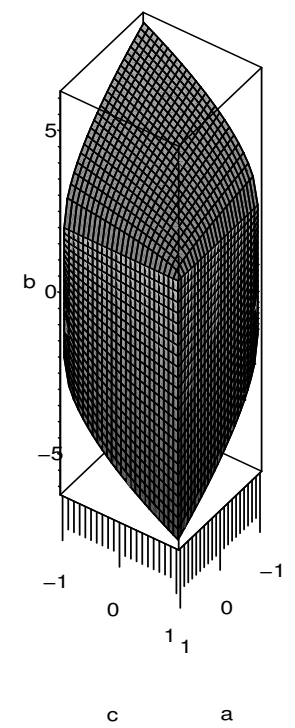

Figure 1: Unit ball of $\left(\mathbb{R}^{3},\|\cdot\|_{\Delta}\right)$.

(a) $\mathrm{S}_{\Delta}=\operatorname{graph}\left(\left.f_{+}\right|_{\mathrm{B}_{\ell_{\infty}^{2}}}\right) \cup \operatorname{graph}\left(\left.f_{-}\right|_{\mathrm{B}_{\ell_{\infty}^{2}}}\right) \cup F$.

(b) $\operatorname{ext}\left(\mathrm{B}_{\Delta}\right)=\{ \pm(1,-2-2 \sqrt{2(1+t)}, t), \pm(t,-2-2 \sqrt{2(1+t)}, 1): t \in[-1,1]\}$.

Proof. Part (a) can be obtained from Theorems 2.1 and 2.2 through standard calculations. As for part (b), notice that for every $\left(a_{0}, b_{0}, c_{0}\right) \in F$ such that $f_{-}\left(a_{0}, c_{0}\right)<b_{0}<f_{+}\left(a_{0}, c_{0}\right)$, the point $\left(a_{0}, b_{0}, c_{0}\right)$ is in the interior of the segment $\left\{(a, b, c) \in \mathbb{R}^{3}:(a, c) \in \mathrm{S}_{\ell_{\infty}^{2}}\right.$ and $\left.f_{-}\left(a_{0}, c_{0}\right) \leq b \leq f_{+}\left(a_{0}, c_{0}\right)\right\} \subseteq F$. In addition to that, for every $\left(a_{0}, c_{0}\right) \in \mathrm{B}_{\ell_{\infty}^{2}}$, the mapping $f_{+}$(respectively $f_{-}$), is affine on the straight line given by the equation $\left(1-c_{0}\right) a=\left(1-a_{0}\right) c+a_{0}-c_{0}$ (respectively $\left.\left(1+c_{0}\right) a=\left(1+a_{0}\right) c-a_{0}+c_{0}\right)$. Therefore the extreme points of $\mathrm{B}_{\Delta}$ can occur only at the points where the sets graph $\left(\left.f_{+}\right|_{\mathrm{B}_{\ell_{\infty}^{2}}}\right), \operatorname{graph}\left(\left.f_{-}\right|_{\mathrm{B}_{\ell_{\infty}^{2}}}\right)$ and $F$ meet along a non affine curve. The reader can check easily that this happens only at the points

$$
\pm(1,-2-2 \sqrt{2(1+t)}, t) \text { and } \pm(t,-2-2 \sqrt{2(1+t)}, 1),
$$

where $t \in[-1,1]$.

Figure 1 shows what the unit ball of $\left(\mathbb{R}^{3},\|\cdot\|_{\Delta}\right)$ looks like.

\section{Bernstein and Markov Inequalities in $\mathcal{P}\left({ }^{2} \Delta\right)$}

Bernstein's Inequality (and Markov's Inequality) in $\mathcal{P}\left({ }^{2} \Delta\right)$ is about the size, i.e. norm, of the linear functional $D P(x, y)$, i.e., the sup of the values attained over the set $B_{\ell_{2}^{2}}$, for every given $(x, y) \in \Delta$ (or uniformly for all $(x, y) \in \Delta$ ). It 
can also be expressed as the Euclidean norm of the gradient vector $\nabla P(x, y):=$ $\left(\frac{\partial}{\partial x} P(x, y), \frac{\partial}{\partial y} P(x, y)\right)$.

Theorem 3.1 If $P \in \mathcal{P}\left({ }^{2} \Delta\right)$ then we have

$$
\|D P(x, y)\|_{2} \leq 2 \sqrt{10 x^{2}-12 x y+10 y^{2}} \cdot\|P\|_{\Delta},
$$

for every $(x, y) \in \Delta$. Moreover equality occurs for the polynomials

$$
\pm\left(x^{2}-6 x y+y^{2}\right) \text {. }
$$

Proof. To derive a Bernstein type inequality we first fix $(x, y) \in \Delta$. Let us observe that normalizing by $\|P\|_{\Delta}$, we can deal without loss of generality with polynomials of norm less than or equal to 1 . Therefore, since $\|D P(x, y)\|$ is just the Euclidean norm of the gradient $\nabla P(x, y)$, our task reduces to find

$$
\max \left\{\|\nabla P(x, y)\|_{2}: P \in \mathrm{B}_{\Delta}\right\} .
$$

Notice that, since $\mathrm{B}_{\mathcal{P}\left({ }^{2} \Delta\right)} \ni P \mapsto\|\nabla P(x, y)\|_{2}$ is convex,

$$
\begin{aligned}
\max _{P \in \mathrm{B}_{\Delta}}\|\nabla P(x, y)\|_{2} & =\max _{P \in \operatorname{ext}\left(\mathrm{B}_{\Delta}\right)}\|\nabla P(x, y)\|_{2} \\
& =\max _{-1 \leq t \leq 1}\|(2 a(t) x+b(t) y, b(t) x+2 c(t) y)\|_{2},
\end{aligned}
$$

where $a(t), b(t)$ and $c(t)$ are the coefficients of the extreme polynomials in $\mathrm{B}_{\Delta}$, that (according to part (b) in Theorem 2.3) are given by

$$
\operatorname{ext}\left(\mathrm{B}_{\Delta}\right)=\{ \pm(1,-f(t), t), \pm(t,-f(t), 1): t \in[-1,1]\},
$$

with $f(t)=2+2 \sqrt{2(1+t)}$ for all $t \in[-1,1]$. Observe that the change of $a(t)$ and $c(t)$ bears the same effect as changing the role of $x$ and $y$. Hence we obtain

$$
\left(\max _{P \in \mathrm{B}_{\Delta}}\|\nabla P(x, y)\|\right)^{2}=\max \{h(x, y), h(y, x)\},
$$

where

$$
\begin{aligned}
h(x, y)=\max _{|t| \leq 1}\left[4+(2+2 \sqrt{2(1+t)})^{2}\right] x^{2} & -[4(1+t)(2+2 \sqrt{2(1+t)})] x y \\
+ & {\left[4 t^{2}+(2+2 \sqrt{2(1+t)})^{2}\right] y^{2} . }
\end{aligned}
$$

Here we can assume that $x+y=1$, for homogeneity ensures $\|\nabla P(x, y)\|=$ $|x+y| \cdot\left\|\nabla P\left(\frac{x}{x+y}, \frac{y}{x+y}\right)\right\|$, and extremalizing on $x+y=1$ implies extremals for 
all $(x, y)$. Further, let us put $u:=\sqrt{2(1+t)} \in[0,2]$. Therefore, writing in $u$ and $y=1-x$, we are to consider the maximum of

$$
\begin{aligned}
& \Phi(x, u) \\
& :=\left[4+(2+2 u)^{2}\right] x^{2}-2 u^{2}(2+2 u) x(1-x)+\left[\left(u^{2}-2\right)^{2}+(2+2 u)^{2}\right](1-x)^{2} \\
& =\left(4 u^{2}+8 u+8\right) x^{2}-\left(4 u^{3}+4 u^{2}\right) x(1-x)+\left[u^{4}+8 u+8\right](1-x)^{2} \\
& =\left[u^{4}+4 u^{3}+8 u^{2}+16 u+16\right] x^{2} \\
& \quad+\left[-2 u^{4}+4 u^{3}+4 u^{2}-16 u-16\right] x+\left[u^{4}+8 u+8\right]
\end{aligned}
$$

with fixed $x \in[0,1]$ over the possible values $0 \leq u \leq 2$. Differentiating with respect to $u$ yields

$$
\begin{aligned}
\frac{\partial}{\partial u} \Phi(x, u) & =\left[4 u^{3}+12 u^{2}+16 u+16\right] x^{2}+\left[-8 u^{3}+12 u^{2}+8 u-16\right] x+\left[4 u^{3}+8\right] \\
& =4 u^{3}(x-1)^{2}+12 u^{2}\left(x^{2}+x\right)+8 u(2 x+1)+8 x^{2}+8(x-1)^{2}>0 .
\end{aligned}
$$

Therefore, we find that the required maximum is attained for $u=2$, that is, if $t=1$.

The latter also shows that equality in (3) is attained only for the polynomials $\pm P_{0}$, where $P_{0}(x, y)=x^{2}-6 x y+y^{2}$. Clearly, the change of $x \leftrightarrow y$ leaves the above assertion invariant, since $P_{0}(x, y)=P_{0}(y, x)$. Therefore, in general, we have

$$
\begin{aligned}
\max _{P \in \mathrm{B}_{\Delta}}\|\nabla P(x, y)\|_{2} & =|x+y| \cdot\left\|\left(\nabla P_{0}\right)\left(\frac{x}{x+y}, \frac{y}{x+y}\right)\right\|_{2} \\
& =2 \sqrt{(x-3 y)^{2}+(3 x-y)^{2}} \\
& =2 \sqrt{10 x^{2}-12 x y+10 y^{2}} .
\end{aligned}
$$

This finishes the proof.

For the Markov inequality we need to maximize this quantity over $\Delta$. An easy calculation - or a straightforward reference to convexity and the fact that $\Delta=$ co $\{(0,0),(0,1),(1,0)\}$ - yields that the maximum is reached when $(x, y)=(0,1)$ or $(1,0)$, and then the Bernstein-Markov factor is $2 \sqrt{10}$. Thus we obtain

Corollary 3.2 Let $P \in \mathcal{P}\left({ }^{2} \Delta\right)$ be arbitrary. Then for any $(x, y) \in \Delta$ we have

$$
\|D P(x, y)\|_{2} \leq 2 \sqrt{10} \cdot\|P\|_{\Delta},
$$

and equality occurs for the polynomial $\pm\left(x^{2}-6 x y+y^{2}\right)$ and at the points $(0,1)$ and $(1,0)$.

\section{Polarization constant of $\mathcal{P}\left({ }^{2} \Delta\right)$.}

First we find a pointwise gradient estimate on the whole plane $\mathbb{R}^{2}$ for polynomials in $\mathcal{P}\left({ }^{2} \Delta\right)$ - so, in a sense a Bernstein type inequality - but considering the sup norm over $\Delta$ for the gradient. This inequality, restricted to $\Delta$, will provide in Corollary 4.2 a sharp Markov type inequality for polynomials on $\Delta$ and the polarization constant of the space $\mathcal{P}\left({ }^{2} \Delta\right)$. The latter will be used to show that Martin's inequality (1) does not hold with the same constant when working on non symmetric convex bodies. 
Theorem 4.1 If for each $(x, y) \in \mathbb{R}^{2}, \Psi_{\Delta}(x, y)$ represents the best constant in

$$
\|D P(x, y)\|_{\Delta} \leq \Psi_{\Delta}(x, y)\|P\|_{\Delta}, \quad \text { for every } P \in \mathcal{P}\left({ }^{2} \Delta\right)
$$

then

$$
\Psi_{\Delta}(x, y)= \begin{cases}|2 x-6 y| & \text { if } x=0 \text { or } x \neq 0 \text { and }\left(\frac{y}{x} \leq-1 \text { or } \frac{y}{x} \geq 2\right), \\ \left|2 x+2 y+y^{2} / x\right| & \text { if } x \neq 0 \text { and } 1 \leq \frac{y}{x} \leq 2, \\ \left|2 x+2 y+x^{2} / y\right| & \text { if } y \neq 0 \text { and } 1 \leq \frac{x}{y} \leq 2, \\ |6 x-2 y| & \text { if } y=0 \text { or } y \neq 0 \text { and }\left(\frac{x}{y} \leq-1 \text { or } \frac{x}{y} \geq 2\right) .\end{cases}
$$

Proof. Let us observe that dividing (4) by $\|P\|_{\Delta}$, we can deal without loss of generality with polynomials of norm less than or equal to 1 . Therefore the proof of (4) reduces to finding

$$
\sup \left\{\|\nabla P(x, y)\|_{\Delta}: P \in \mathrm{B}_{\Delta}\right\} .
$$

Notice that if $P(\xi, \zeta)=a \xi^{2}+b \xi \zeta+c \zeta^{2}$ for every $(\xi, \zeta) \in \mathbb{R}^{2}$ with $a, b, c \in \mathbb{R}$, then for a fixed $(x, y) \in \mathbb{R}^{2}$ the mapping

$$
\mathbb{R}^{2} \ni(h, k) \mapsto|\nabla P(x, y) \cdot(h, k)|=|(2 a x+b y) h+(b x+2 c y) k|
$$

is convex and $\Delta$ is a convex body. Therefore $\|\nabla P(x, y)\|_{\Delta}$ is attained at $(0,0),(1,0)$ or $(0,1)$. This lets us obtain the following:

$$
\begin{aligned}
\|\nabla P(x, y)\|_{\Delta} & =\sup \{|(2 a x+b y) h+(b x+2 c y) k|:(h, k) \in \Delta\} \\
& =\max \{|2 a x+b y|,|b x+2 c y|\} .
\end{aligned}
$$

Note that this formula also shows that $\Psi_{\Delta}(x, y)$ is a convex function of $(x, y) \in \mathbb{R}^{2}$, as it is represented as the maximum of the above convex function of $(x, y) \in \mathbb{R}^{2}$ over $(a, b, c)$ corresponding to $\mathrm{B}_{\Delta}$.

Now since $\mathrm{B}_{\Delta}$ is a convex body and the mapping $\mathrm{B}_{\Delta} \ni P \mapsto\|\nabla P(x, y)\|_{\Delta}$ is convex, we have that

$$
\Psi_{\Delta}(x, y)=\sup \left\{\|\nabla P(x, y)\|_{\Delta}: P \in \operatorname{ext}\left(\mathrm{B}_{\Delta}\right)\right\} .
$$

On the other hand, according to part (b) in Theorem 2.3,

$$
\operatorname{ext}\left(\mathrm{B}_{\Delta}\right)=\{ \pm(1,-f(t), t), \pm(t,-f(t), 1): t \in[-1,1]\}
$$

where $f(t)=2+2 \sqrt{2(1+t)}$ for all $t \in[-1,1]$. Hence we obtain

$$
\begin{aligned}
\Psi_{\Delta}(x, y) & =\sup _{s, t \in[-1,1]}\{|2 x-f(t) y|,|-f(t) x+2 t y|,|2 t x-f(t) y|,|-f(t) x+2 y|\} \\
& =\max \left\{\left\|F_{k}^{x y}\right\|: k=1,2,3,4\right\}
\end{aligned}
$$

where $F_{1}^{x y}(t):=2 x-f(t) y, F_{2}^{x y}(t):=-f(t) x+2 t y, F_{3}^{x y}(t):=2 t x-f(t) y$ and $F_{4}^{x y}(t):=-f(t) x+2 y$, for every $t \in[-1,1]$ and $\left\|F_{k}^{x y}\right\|:=\sup \left\{\left|F_{k}^{x y}(t)\right|:-1 \leq t \leq 1\right\}$ for $k=1,2,3,4$. 
Now let us consider the case when $x=0$. Then

$$
\Psi_{\Delta}(0, y)=\max _{t \in[-1,1]}\{|f(t) y|,|2 t y|,|2 y|\}=\max _{t \in[-1,1]}\{|f(t)|, 2|t|, 2\}|y|=6|y| .
$$

Similarly, if $y=0$, then we obtain $\Psi_{\Delta}(x, 0)=6|x|$.

This also follows from the obvious invariance with respect to the change of variables $(x, y) \leftrightarrow(y, x)$, which must leave $\Psi_{\Delta}$ unchanged in view of the symmetry of $\Delta$ with respect to the line $y=x$. There is a further obvious symmetry, namely $\Psi_{\Delta}(-x,-y)=\Psi_{\Delta}(x, y)$. Therefore, for the rest of the proof we may assume $x y \neq 0$ and $y>0$. Hence it suffices to restrict to two further cases, Case I being $0<x \leq y$ and Case II being $0<-x \leq y$.

The mapping $F_{1}^{x y}$ is monotone, and therefore

$$
\left\|F_{1}^{x y}\right\|=\max \left\{\left|F_{1}^{x y}(-1)\right|,\left|F_{1}^{x y}(1)\right|\right\} .
$$

On the other hand $F_{2}^{x y}$ has a unique critical point at the root of the equation

$$
\frac{-2}{\sqrt{2(1+t)}} x+2 y=0
$$

i.e. when $(0 \leq) \sqrt{2(1+t)}=x / y$. Clearly such a critical point $t_{x y}$ exists only in Case I, since in Case II $x / y<0$. Furthermore, $t_{x y}=\frac{x^{2}-2 y^{2}}{2 y^{2}} \in[-1,1]$ if and only if $x / y \in[0,2]$, which is evident in view of $y \geq x>0$. Hence

$$
\left\|F_{2}^{x y}\right\|= \begin{cases}\max \left\{\left|F_{2}^{x y}(-1)\right|,\left|F_{2}^{x y}(1)\right|,\left|F_{2}^{x y}\left(t_{x y}\right)\right|\right\} & \text { if } 0<x \leq y \\ \max \left\{\left|F_{2}^{x y}(-1)\right|,\left|F_{2}^{x y}(1)\right|\right\} & \text { if } 0<-x \leq y .\end{cases}
$$

Now notice that $F_{4}^{x y} \equiv F_{1}^{y x}$ and that $F_{3}^{x y} \equiv F_{2}^{y x}$. Therefore

$$
\left\|F_{3}^{x y}\right\|= \begin{cases}\max \left\{\left|F_{2}^{y x}(-1)\right|,\left|F_{2}^{y x}(1)\right|,\left|F_{2}^{y x}\left(t_{y x}\right)\right|\right\} & \text { if } 0<x \leq y \leq 2 x, \\ \max \left\{\left|F_{2}^{y x}(-1)\right|,\left|F_{2}^{y x}(1)\right|\right\} & \text { if } 0<-x \leq y \text { or } 0<x<y / 2 .\end{cases}
$$

and

$$
\left\|F_{4}^{x y}\right\|=\max \left\{\left|F_{1}^{y x}(-1)\right|,\left|F_{1}^{y x}(1)\right|\right\} .
$$

Let us introduce the auxiliary functions

$$
\begin{aligned}
\Theta_{1}(x, y) & :=\max \left\{\left|F_{1}^{x y}(-1)\right|,\left|F_{1}^{x y}(1)\right|,\left|F_{1}^{y x}(-1)\right|,\left|F_{1}^{y x}(1)\right|\right\} \\
& =\max \{|2 x-2 y|,|2 x-6 y|,|2 y-2 x|,|2 y-6 x|\}, \\
\Theta_{2}(x, y) & :=\max \left\{\left|F_{2}^{x y}(-1)\right|,\left|F_{2}^{x y}(1)\right|,\left|F_{2}^{y x}(-1)\right|,\left|F_{2}^{y x}(1)\right|\right\} \\
& =\max \{|-2 x-2 y|,|-6 x+2 y|,|-2 y-2 x|,|-6 y+2 x|\}, \\
\Theta(x, y) & :=\max \left\{\Theta_{1}(x, y), \Theta_{2}(x, y)\right\} .
\end{aligned}
$$

Then, by the latter, we have

$$
\Psi_{\Delta}(x, y)= \begin{cases}\max \left\{\Theta(x, y),\left|F_{2}^{x y}\left(t_{x y}\right)\right|,\left|F_{2}^{y x}\left(t_{y x}\right)\right|\right\} & \text { if } 0<x \leq y \leq 2 x \\ \max \left\{\Theta(x, y),\left|F_{2}^{x y}\left(t_{x y}\right)\right|\right\} & \text { if } 0<x<y / 2 \\ \Theta(x, y) & \text { if } 0<-x \leq y\end{cases}
$$




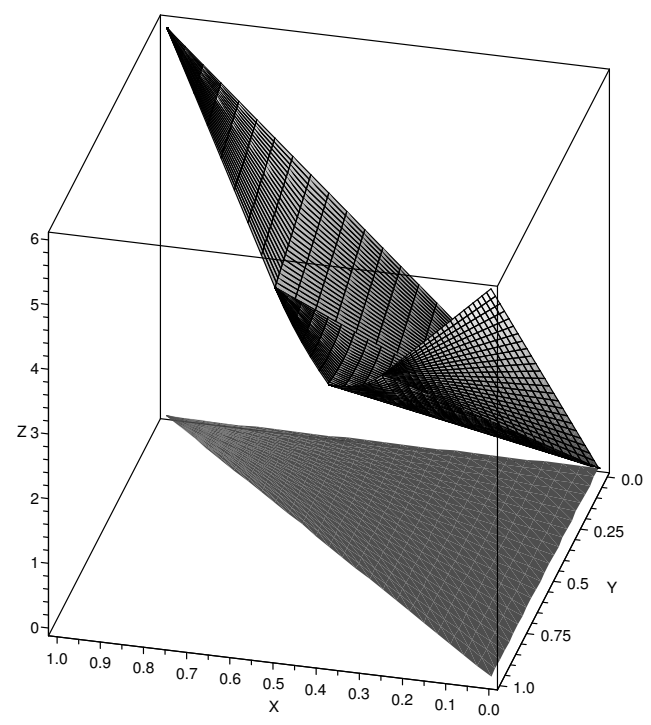

Figure 2: $\Psi_{\Delta}(x, y)$ on the simplex.

Since now $0<|x| \leq y$, it is easy to see that $\Theta_{1}(x, y)=6 y-2 x$ and also that $\Theta_{2}(x, y)=6 y-2 x$, hence $\Theta(x, y)=6 y-2 x$, too. On the other hand in Case I we have $\left|F_{2}^{x y}\left(t_{x y}\right)\right|=2 y+2 x+x^{2} / y$ and $\left|F_{2}^{y x}\left(t_{y x}\right)\right|=2 x+2 y+y^{2} / x$. Therefore, $F_{2}^{y x}\left(t_{y x}\right)|\geq| F_{2}^{x y}\left(t_{x y}\right) \mid$, and we arrive at

$$
\Psi_{\Delta}(x, y)= \begin{cases}\max \left\{6 y-2 x, 2 x+2 y+y^{2} / x\right\} & \text { if } 0<x \leq y \leq 2 x \\ \max \left\{6 y-2 x, 2 x+2 y+x^{2} / y\right\} & \text { if } 0<x<y / 2 \\ 6 y-2 x & \text { if } 0<-x \leq y\end{cases}
$$

Consider first the domain where $6 y-2 x \leq 2 x+2 y+x^{2} / y$, i.e. where $x^{2}+$ $4 y x-4 y^{2} \geq 0$. In the positive quadrant the solutions are the points satisfying $x \geq(2 \sqrt{2}-2) y$, which is disjoint from the domain $0<x<y / 2$, thus proving that in the second case of $(5)$, we also have $\Psi_{\Delta}(x, y)=6 y-2 x$. In the first case of (5) $6 y-2 x \leq 2 x+2 y+y^{2} / x$ is equivalent to $(2 x-y)^{2} \geq 0$, holding for all $0<x \leq y$. Therefore, we are led to

$$
\Psi_{\Delta}(x, y)= \begin{cases}2 x+2 y+y^{2} / x & \text { if } 0<x \leq y \leq 2 x \\ 6 y-2 x & \text { if } 0<x<y / 2 \text { or } 0<-x \leq y .\end{cases}
$$

The reader can check that the above results, valid for the quadrant $0<|x| \leq y$, carry over to the other points of $\mathbb{R}^{2}$ as stated in the formulation of the theorem.

As a consequence of the previous result we can establish the following Markov type estimate for polynomials in $\mathcal{P}\left({ }^{2} \Delta\right)$. 
Corollary 4.2 If $P \in \mathcal{P}\left({ }^{2} \Delta\right)$ then

$$
\max _{(x, y) \in \Delta}\|D P(x, y)\| \leq 6\|P\|_{\Delta} .
$$

Furthermore, 6 is optimal in (7) since equality holds for the polynomial $P(x, y)=$ $x^{2}-6 x y+y^{2}$.

Proof. According to Theorem 4.1, we just need to maximize $\Psi_{\Delta}$ over the simplex $\Delta$. Notice that $\Psi_{\Delta}$ is a convex mapping on $\Delta$, as is established in the course of proof of Theorem 4.1. Therefore:

$$
\begin{aligned}
\|D P\|_{\Delta} & \leq \max _{(x, y) \in \Delta} \Psi_{\Delta}(x, y)\|P\|_{\Delta} \\
& =\max \left\{\Psi_{\Delta}(0,0), \Psi_{\Delta}(0,1), \Psi_{\Delta}(1,0)\right\}\|P\|_{\Delta} \\
& =6\|P\|_{\Delta} .
\end{aligned}
$$

Remark 4.3 If $P \in \mathcal{P}\left({ }^{2} \Delta\right)$, then $D P(\mathbf{x})=2 \check{P}(\mathbf{x}, \cdot)$ for every $\mathbf{x} \in \mathbb{R}^{2}$. This shows that $\|D P(\mathbf{x})\|_{\Delta}=2\|\check{P}\|_{\Delta}$ for all $\mathbf{x} \in \mathbb{R}^{2}$ and, hence using $(7)$ we derive

$$
\|\check{P}\|_{\Delta} \leq 3\|P\|_{\Delta}
$$

Furthermore, as the constant 3 here is sharp in view of Corollary 4.2, Martin's inequality (1) does not hold for polynomials on a non symmetric convex body.

\section{References}

[1] R. M. Aron And M. Klimek. Supremum norms for quadratic polynomials. Arch. Math. (Basel) 76 (2001), 73-80.

[2] M. BARAn. Bernstein type theorems for compact sets in $\mathbb{R}^{n}$ revisited. J. Approx. Th. 79(1994), 190-198.

[3] D. Burns, N. Levenberg, S. MA'u, Sz. RÉvész. Monge-Ampère Measures for Convex Bodies and Bernstein-Markov Type Inequalities, arXiv:0705.1095v1.

[4] N. G. Chebotarëv. On a general criterion of the minimax, C. R. (Doklady) Acad. Sci. URSS (N.S.), 39(1943), p. 341.

[5] Y. S. Choi And S. G. KIm. The unit ball of $\mathcal{P}\left({ }^{2} l_{2}^{2}\right)$. Arch. Math. (Basel) 76 (1998), 472-480.

[6] Y. S. Choi And S. G. Kim. Smooth points of the unit ball of the space $\mathcal{P}\left({ }^{2} l_{1}\right)$. Results Math. 36 (1999), 26-33.

[7] Y. S. Choi And S. G. Kim. Exposed points of the unit balls of the spaces $\mathcal{P}\left({ }^{2} l_{p}^{2}\right)(p=1,2, \infty)$. Indian J. Pure Appl. Math. 35 (2004), 37-41. 
[8] B. C. Grecu. Geometry of homogeneous polynomials on two-dimensional real Hilbert spaces. J. Math. Anal. Appl. 293 (2004), no. 1, 578-588.

[9] B. C. Grecu. Extreme 2-homogeneous polynomials on Hilbert spaces. Quaest. Math. 25 (2002), no. 4, 421-435.

[10] B. C. Grecu. Geometry of 2-homogeneous polynomials on $l_{p}$ spaces, $1<p<$ $\infty$. J. Math. Anal. Appl. 273 (2002), no. 1, 262-282.

[11] B. C. Grecu. Smooth 2-homogeneous polynomials on Hilbert spaces. Arch. Math. (Basel) 76 (2001), no. 6, 445-454.

[12] B. C. Grecu. Geometry of three-homogeneous polynomials on real Hilbert spaces. J. Math. Anal. Appl. 246 (2000), no. 1, 217-229.

[13] B. C. Grecu, G. A. Muñoz-Fernández and J. B. Seonne-Sepúlveda. The unit ball of the complex $\mathcal{P}\left({ }^{3} H\right)$. Preprint, 2007.

[14] L. A. HARRIS, Multivariate Markov polynomial inequalities and Chebyshev nodes J. Math. Anal. Appl. 338, no. 1, February 2008, pp. 350-357.

[15] G. KlimeK And M. KlimeK. Discovering curves and surfaces with Maple. New York, 1997.

[16] A. G. Konheim And T. J. Rivlin, Extreme points of the unit ball in a space of real polynomials. Amer. Math. Monthly 73 (1966), 505-507.

[17] R. S. Martin. Ph. D. Thesis. Cal. Inst. of Tech. 1932.

[18] L. B. Milev, Sz. Gy. RÉvÉsz, Bernstein's inequality for multivariate polynomials on the standard simplex, J. Inequalities Appl., 2005:2 (2005), 145-163.

[19] G. A. Muñoz-Fernández and Y. SARAntopoulos. Bernstein and Markov-type inequalities for polynomials on real Banach spaces. Math. Proc. Camb. Phil. Soc. 133(2002), 515-530.

[20] G. A. Muñoz-Fernández and J. B. Seonne-Sepúlveda. Geometry of Banach spaces of Trinomials. J. Math. Anal. Appl. 340 (2008), 1069-1087.

[21] G. A. Muñoz-Fernández, Y. Sarantopoulos and J. B. SeonneSepúlvedA. An application of the Krein-Milman Theorem to Bernstein and Markov inequalities. To appear in J. Convex Anal.

[22] D. Nadzhmiddinov and Yu. N. Subbotin. Markov inequalities for polynomials on triangles. (Russian) Mat. Zametki 46 (1989), 2, 76-82, 159; translation in Math. Notes 46 (1989), 1-2, 627-631.

[23] S. Neuwirth. The maximum modulus of a trigonometric trinomial. arXiv:math/FA.0703236v1. 
[24] S. RÉvÉSz. Minimization of maxima of nonnegative and positive definite cosine polynomials with prescribed first coefficients. Acta Sci. Math. (Szeged), 60 (1995), 589-608.

[25] Y. Sarantopoulos. Bounds on the derivatives of polynomials on Banach spaces. Math. Proc. Camb. Phil. Soc. 110(1991), 307-312.

[26] V. I Skalyga, Bounds on the derivatives of polynomials on entrally symmetric convex bodies, (Russian), Izv. Ross. Akad. Nauk Ser. Mat. 69 (2005), no. 3, 179192; translation in Izv. Math. 69 (2005), no. 3, 607621.

[27] E.V. Voronovskaya. The functional method and its applications. Appendix: V.A. Gusev: Derivative functionals of an algebraic polynomial and V. A. Markov's theorem. American Mathematical Society (AMS) VI 203 (1970).

\section{G. A. Muñoz-Fernández and J. B. Seoane-Sepúlveda.}

Facultad de Ciencias Matemáticas. Departamento de Análisis Matemático.

Universidad Complutense de Madrid. Plaza de las Ciencias 3. 28040. Madrid. Spain.

Emails: gustavo_fernandez@mat.ucm.es and jseoane@mat.ucm.es

\section{Szilárd Gy. Révész.}

Renyi Institute of Mathematics, Hungarian Academy of Sciences. Budapest.

Hungary.

Email: revesz@renyi.hu 